\title{
SEGMENTATION OF FETAL 3D ULTRASOUND BASED ON STATISTICAL PRIOR AND DEFORMABLE MODEL
}

\author{
Jérémie Anquez, Elsa D. Angelini, Isabelle Bloch \\ Institut TELECOM, TELECOM ParisTech, LTCI CNRS, Paris, France.
}

\begin{abstract}
A statistical variational framework is proposed for the fetus and uterus segmentation in ultrasound images. The Rayleigh and exponential distributions are used to model the pixel intensity. An energy is derived to perform an optimal partition of the $3 \mathrm{D}$ data into two classes corresponding to these two distributions, in a Bayesian MAP framework. Some numerical difficulties are raised by the combination of heterogeneous distributions in a variational level-set formulation, as discussed in the paper. Results on simulated and real data are presented and show that assuming different distributions provides better results than with the sole Rayleigh distribution.
\end{abstract}

Index Terms - 3D ultrasound, segmentation, deformable model, statistical prior

\section{INTRODUCTION}

Ultrasounds imaging (echography) is the main imaging modality used for pregnancy follow up [1], combining several advantages such as being portable, low-cost, non invasive and non ionizing. In order to follow the fetus development, several biometric measurements are extracted from ultrasound data. Recent 3D ultrasound systems enable volume measurements and provide useful additional information. A segmentation process is desirable to automate these measures. In order to segment the fetus and the uterus, it is possible to take advantage of the amniotic fluid filling the uterus and surrounding the fetus. Distinguishing the maternal and fetal tissues from the amniotic fluid, a segmentation of both the fetus and uterus could be performed.

Different approaches have been developed to detect structures on ultrasound data [2]. Some methods are based on the statistical properties of the tissues gray level intensity usually modeled with Rayleigh distribution. After manually segmenting several datasets, we computed the gray level intensity histograms of two different pixel classes: the fetal and maternal tissues pixels on the one hand, the amniotic fluid pixels on the other hand. The Rayleigh distribution fits well the histogram of the pixels intensity in the fetal and maternal tissues. However, this distribution model is not suitable when we consider the amniotic fluid and the exponential distribution provides a more appropriate representation of the pixels intensity distribution.

In this paper, we propose a deformable model formulation to partition the image data based on the gray level statistical distributions of the structures to identify. The Rayleigh distribution is used to represent the intensity of the pixels belonging to the fetal and maternal tissues while the exponential distribution is used for the the

This work was partially funded by Orange Labs R\&D and Fondation Santé et Radiofréquences (FEMONUM project). The authors thank Philips Healthcare Research Laboratories, Paris, France, for providing the 3D ultrasound data. pixels belonging to the amniotic fluid. First, we present a framework embedding this statistical prior. Then, an energy is defined and derived to obtain the corresponding Euler-Lagrange equations. The associated flow is determined using level-sets. Finally, we present experimental results obtained on simulated and real data.

\section{INTRODUCING STATISTICAL DISTRIBUTIONS OF GRAY LEVEL INTO A LEVEL-SETS FRAMEWORK}

Let $\Omega$ be a bounded and open subset of $\mathbb{R}^{N}$ and $I: \Omega \rightarrow \mathbb{R}$ an image. A given closed curve $C$ in $\Omega$ defines a partition of the image domain in an inside region $\Omega_{i}$ and an outside region $\Omega_{e}$. The proposed segmentation method relies on a region-based approach, embedding an a priori statistical knowledge of the distribution of the pixels intensity within each region. Let $\Omega_{i}$ (resp. $\Omega_{e}$ ) be the region corresponding to the pixels belonging to the fetal and maternal tissues (resp. the amniotic fluid). The intensity of the pixels belonging to $\Omega_{i}$ (resp. $\Omega_{e}$ ) is modeled with a random variable $I_{i}$ (resp. $I_{e}$ ) following a Rayleigh distribution (resp. an exponential distribution). We look for the curve $C$ providing a maximum a posteriori segmentation in a Bayesian framework with respect to the probability distributions of the image pixels, $P(C \mid I)=P(C) P(I \mid C)$.

We model $P(C)$ using an exponential distribution with parameter $\mu>0$. We have $p(C=c)=\mu \exp (-\mu|c|)$, with $|c|$ the curve length. $p(C=c)$ is a decreasing function of $|c|$. Hence, smooth curves are favored as $|c|$ increases with local oscillations. Let $|\bar{C}|$ the mean of the curve length. We have $|\bar{C}|=1 / \mu$. $|\bar{C}|$ being a decreasing function of $\mu$, the value of $\mu$ defines the regularization strength. The higher value of $\mu$, the more regularized $C$.

Let $f(I(x))$ be the pixels intensity probability density function (pdf). We have $p(I=I(x))=f(I(x))$. As the pixel intensity depends on the image partition defined by $C$, we get:

$$
p(I=I(x) \mid C)=\left\{\begin{array}{l}
p\left(I_{i}=I(x) \mid x \in \Omega_{i}\right)=f_{i}\left(I(x), \sigma_{i}\right) \\
\text { if } x \in \Omega_{i} \\
p\left(I_{e}=I(x) \mid x \in \Omega_{e}\right)=f_{e}\left(I(x), \lambda_{e}\right) \\
\text { if } x \in \Omega_{e},
\end{array}\right.
$$

with,

$$
\left\{\begin{array}{l}
f_{i}\left(I(x), \sigma_{i}\right)=\frac{I(x)}{\sigma_{i}^{2}} \exp \left(-\frac{I(x)^{2}}{2 \sigma_{i}^{2}}\right) \\
f_{e}\left(I(x), \lambda_{e}\right)=\lambda_{e} \exp \left(-\lambda_{e} I(x)\right)
\end{array}\right.
$$

Pixels intensities are assumed to be uncorrelated and independently distributed, conditionally to $C$. Let $P_{i}=\prod_{x \in \Omega_{i}} f_{i}\left(I(x), \sigma_{i}\right)$ (resp. $\left.P_{e}=\prod_{x \in \Omega_{e}} f_{e}\left(I(x), \lambda_{e}\right)\right)$ be the joint probability of the pixels intensities inside (resp. outside) the curve. We have $P(I \mid C)=$ $P_{i} P_{e}$, and thus $P(C \mid I)=P(C) P_{i} P_{e}$. Our goal is to identify the curve $C$ maximizing this quantity. We use the logarithm to linearize the product and set $l\left(I, C, \sigma_{i}, \lambda_{e}\right)=\log (P(C \mid I))$. The maximum 
value of $P(C \mid I)$ occurs at the same points as the maximum value of $l$.

We have:

$$
\begin{gathered}
l\left(I, C, \lambda_{e}, \sigma_{i}\right)=l_{\text {reg }}(C)+l_{i}\left(I, C, \sigma_{i}\right)+l_{e}\left(I, C, \lambda_{e}\right), \\
\text { with }\left\{\begin{array}{l}
l_{\text {reg }}(C)=\log \mu-\mu|C| \\
l_{i}\left(I, C, \sigma_{i}\right)=\int_{x \in \Omega_{i}} \log f_{i}\left(I(x), \sigma_{i}\right) \mathrm{d} x \\
l_{e}\left(I, C, \lambda_{e}\right)=\int_{x \in \Omega_{e}} \log f_{e}\left(I(x), \lambda_{e}\right) \mathrm{d} x
\end{array}\right.
\end{gathered}
$$

\subsection{Energy definition}

To obtain the maximum a posteriori segmentation, we define an energy $E=-l$ to be minimized, with $E_{r e g}=-l_{r e g}, E_{i}=-l_{i}$ and $E_{e}=-l_{e}$.

We use a level-set framework and introduce an implicit function $\phi: \Omega \rightarrow \mathbb{R}$ in order to compute the first variation of $E$. The values of $\phi(x)$ are negative inside $\Omega_{i}$ and positive inside $\Omega_{e}$, defining implicitly the curve $C$ as the zero level of the function [3]. E can be rewritten as:

$$
E\left(I, \phi, \sigma_{i}, \lambda_{e}\right)=E_{r e g}(\phi)+E_{i}\left(I, \phi, \sigma_{i}\right)+E_{e}\left(I, \phi, \lambda_{e}\right)
$$

We express $|C|$ in $E_{r e g}(\phi)$ using $\phi$ and $H(\phi)$, the heaviside function [4], and rewrite the $E_{i}$ and $E_{e}$ energy terms as integrals over the entire image using $\phi$ :

$$
\begin{aligned}
& E_{r e g}(\phi)=-\log \mu+\mu \int_{x \in \Omega} \delta(\phi(x))|\nabla \phi(x)| \mathrm{d} x, \\
& E_{i}\left(I, \phi, \sigma_{i}\right)=-\int_{x \in \Omega}(1-H(\phi(x))) \log \left(f_{i}\left(I(x), \sigma_{i}\right)\right) \mathrm{d} x, \\
& E_{e}\left(I, \phi, \lambda_{e}\right)=-\int_{x \in \Omega} H(\phi(x)) \log \left(f_{e}\left(I(x), \lambda_{e}\right)\right) \mathrm{d} x .
\end{aligned}
$$

A similar energy was proposed by [5] where a maximum likelihood segmentation was achieved, using the sole Rayleigh distribution to model pixel intensities in both regions.

\subsection{Energy minimization}

In the following, we minimize the partial derivatives of $E$. The global iterative minimization process consists in alternatively minimizing $E$ in $\sigma_{i}$ and $\lambda_{e}$ (for fixed $\phi$ ) and in $\phi$ (for fixed $\sigma_{i}$ and $\left.\lambda_{e}\right)[6]$.

Firstly, setting to zero $\frac{\partial E}{\partial \sigma_{i}}$ (resp. $\frac{\partial E}{\partial \lambda_{e}}$ ) for fixed $\phi$, we get the value of $\sigma_{i}$ (resp. $\lambda_{e}$ ) minimizing $E$ :

$$
\left\{\begin{aligned}
\sigma_{i} & =\left(\frac{\int_{x \in \Omega}(1-H(\phi(x))) I(x)^{2} \mathrm{~d} x}{2 \int_{x \in \Omega}(1-H(\phi(x))) \mathrm{d} x}\right)^{\frac{1}{2}} \\
\lambda_{e} & =\frac{\int_{x \in \Omega} H(\phi(x)) \mathrm{d} x}{\int_{x \in \Omega} H(\phi(x)) I(x) \mathrm{d} x}
\end{aligned}\right.
$$

Secondly, in order to minimize the energy $E$ with respect to $\phi$, we determine the Euler-Lagrange equation for $\phi$ using the Gâteaux derivative of $E$, keeping $\sigma_{i}$ and $\lambda_{e}$ fixed.

$E$ is differentiable in the Gâteaux sense with respect to $\phi \in X$ if the application $\psi \mapsto E^{\prime}(\phi, \psi)=\lim _{t \rightarrow 0} \frac{1}{t}(E(\phi+t \psi)-E(\phi))$ is defined for any $\psi$ and if it is linear and continuous.

We consider regularized versions $H_{\epsilon}$ and $\delta_{\epsilon}$ of $H$ and $\delta$. After some developments [7], we get:

$$
\begin{aligned}
& E^{\prime}(\psi, \phi)=\int_{\Omega} \delta_{\epsilon}(\phi(x))\left(-\mu \operatorname{div}\left(\frac{\nabla \phi(x)}{|\nabla \phi(x)|}\right)+\log \left(f_{i}\left(I(x), \sigma_{i}\right)\right)\right. \\
& \left.-\log \left(f_{e}\left(I(x), \lambda_{e}\right)\right)\right) \psi \mathrm{d} x+\int_{x \in \partial \Omega} \mu \frac{\delta_{\epsilon}(\phi(x))}{|\nabla \phi(x)|} \frac{\partial \phi(x)}{\partial n} \psi \mathrm{d} x
\end{aligned}
$$

This expression must vanish for all $\psi$ in order to obtain the Euler-Lagrange equation for $\phi$. Therefore, we obtain the following problem:

$$
\begin{aligned}
0=\delta_{\epsilon}(\phi(x))( & \mu \operatorname{div}\left(\frac{\nabla \phi(x)}{|\nabla \phi(x)|}\right) \\
& \left.-\log \left(f_{i}\left(I(x), \sigma_{i}\right)\right)+\log \left(f_{e}\left(I(x), \lambda_{e}\right)\right)\right)
\end{aligned}
$$

with boundary condition:

$$
\frac{\delta_{\epsilon}(\phi(x))}{|\nabla \phi(x)|} \frac{\partial \phi(x)}{\partial n}=0, x \in \partial \Omega .
$$

Putting together (1) and the associated flow to (2), we obtain the system to be solved:

$$
\left\{\begin{array}{l}
\sigma_{i}=\left(\frac{\int_{x \in \Omega}(1-H(\phi(x))) I(x)^{2} \mathrm{~d} x}{2 \int_{x \in \Omega}(1-H(\phi(x))) \mathrm{d} x}\right)^{\frac{1}{2}} \\
\lambda_{e}=\frac{\int_{x \in \Omega} H(\phi(x)) \mathrm{d} x}{\int_{x \in \Omega} H(\phi(x)) I(x) \mathrm{d} x} \\
\frac{\partial \phi}{\partial t}=\delta_{\epsilon}(\phi(x))\left(F_{r e g}+F_{\text {data }}\right) \text { in } \Omega, \\
F_{\text {reg }}=\mu \operatorname{div}\left(\frac{\nabla \phi(x)}{|\nabla \phi(x)|}\right), \\
F_{\text {data }}=-\log \left(f_{i}\left(I(x), \sigma_{i}\right)+\log \left(f_{e}\left(I(x), \lambda_{e}\right),\right.\right. \\
\phi(x, 0)=\phi_{0}(x) \text { in } \Omega, \\
\frac{\delta(\phi)}{|\nabla \phi|} \frac{\partial \phi}{\partial n}=0 \text { on } \partial \Omega .
\end{array}\right.
$$

This system is approximated with finite differences and solved iteratively. In order to reduce the sensitivity of the method to initial conditions, we use an implementation of the $\delta_{\epsilon}$ function allowing $\phi$ to evolve over the entire image domain, even far from its zero level. We use the divergence operator discretization scheme described in [8]. When $\phi$ is updated at a given iteration, the regularization term $F_{\text {reg }}$ and the data fidelity term $F_{\text {data }}$ are evaluated at every pixel of the image domain. The method complexity is the same as in [8].

As illustrated in Figure 2, when the intensity of a pixel belongs more likely to the Rayleigh (resp. exponential) distribution, the value of $F_{d a t a}$ is negative (resp. positive). Considering only $F_{\text {data }}$, $\frac{\partial \phi}{\partial t}$ is consequently negative (resp. positive), and $\phi$ decreases (resp. increases) at this pixel.

\section{EXPERIMENTAL RESULTS}

\subsection{Results on simulated data}

A study was performed on simulated data, to evaluate the segmentation framework. A manually segmentated clinical 3D volume was used to take into account the fetus complex geometry. Two classes of tissues were simulated: the fetal and maternal tissues on the one hand, the amniotic fluid on the other hand. Intensity of the pixels belonging to the former were randomly generated from a Rayleigh distribution of parameter $\sigma$, while the intensity of the pixels of the latter were randomly generated from an exponential distribution of parameter $\lambda$. Different datasets were tested. We present here results obtained on two meaningful samples. In the first case, we used $\sigma=70$ which, together with $\lambda=1 / 30$, corresponds to the parameter evaluated experimentally on real data. In the second case, using $\lambda=1 / 130$, we studied a different situation where the mean intensity of the pixels following the exponential distribution is superior to the mean intensity of the pixels following the Rayleigh distribution. Figure 1 shows the pdfs of the Rayleigh distribution with $\sigma=70$ and of the exponential distributions with $\lambda=\{1 / 30,1 / 130\}$, a slice of the segmented $3 \mathrm{D}$ volume and the corresponding slices in the simulated data. With $\lambda=1 / 30$, the distributions are well differentiated. 
Low (resp. high) intensity pixels follow more likely the exponential (resp. Rayleigh) distribution. We create a more complex case with $\lambda=1 / 130$ where pixels with low and high intensities follow more likely the exponential distribution, while intermediate intensities follow more likely the Rayleigh distribution.
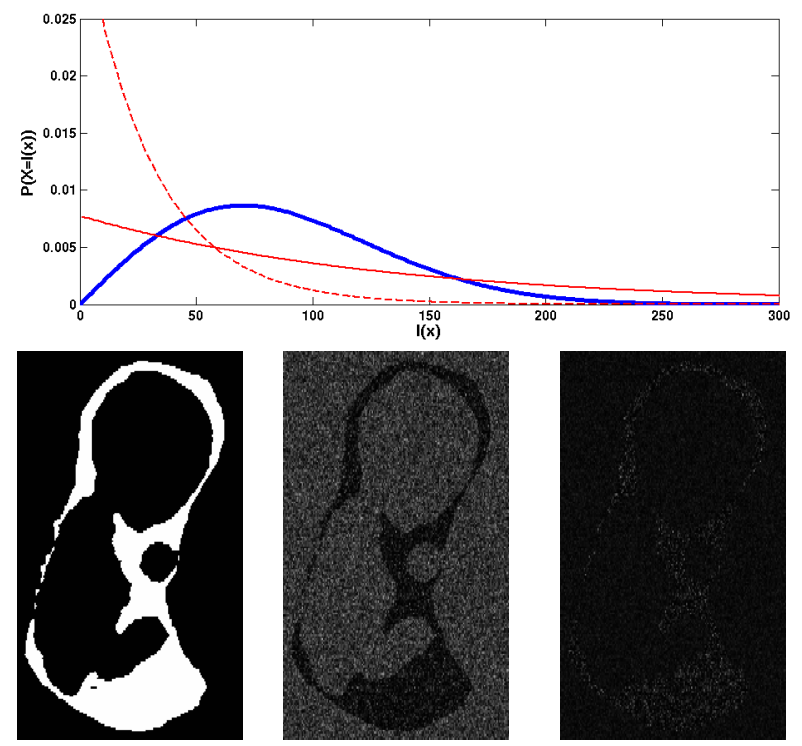

Fig. 1. Top: pdfs of the distributions: $f_{i}(I(x), 70)$ (blue, thick line), $f_{e}(I(x), 1 / 30)$ (red, dotted line) and $f_{e}(I(x), 1 / 130)$ (red, plain line). Bottom: one slice manually segmentated on a 3D ultrasound volume. White pixels belong to the amniotic fluid and black pixels to the fetal and maternal tissues (left). Corresponding slice in two simulated datasets: fetal and maternal tissues generated using a Rayleigh distribution with $\sigma=70$ and the amniotic fluid generated using an exponential distribution with $\lambda=1 / 30$ (center) and $\lambda=1 / 130$ (right).

The value of $\mu$ was set to 1.2 in all experiments. This value provides a good trade off between the geometry of the object to segment and the smoothness of the segmented contours. The $\phi$ function was initialized using cylinders regularly placed in the image. From 1 to 100 cylinders were used for testing purposes and good results were obtained using the different initializations. Figure 2 shows $F_{\text {data }}$ as a function of the intensity $I(x)$ of a pixel $x$ when $\sigma=70$ and $\lambda=1 / 130$. Note that as expected, the zero crossings of $F_{\text {data }}(I(x), 70,1 / 130)$ correspond to the crossings of the distribution pdfs $f_{i}(I(x), 70)$ and $f_{e}(I(x), 1 / 130)$ shown in Figure 1.

Combining two different pdf in $F_{\text {data }}$ raised some numerical difficulties, when evolving the level set function $\phi$. Indeed Figure 2 shows that the maximum value of $\left|F_{\text {data }}\right|$ for $F_{\text {data }}<0$ is five times smaller than the maximum value of $\left|F_{\text {data }}\right|$ for $F_{\text {data }}>0$. Consequently, magnitudes of $\phi$ decreasing variations are lower than magnitudes of $\phi$ increasing variations. Therefore, the $\phi$ function needs to be reinitialized to prevent inappropriate behavior of the numerical approximation of the regularization term. A reinitialization step was performed every 100 iterations. This value has been set experimentally to achieve a good trade off between computation burden and numerical stability.

When the model achieves a good segmentation of the image, it enters an oscillatory state. Firstly, as $\phi$ presents strong variations

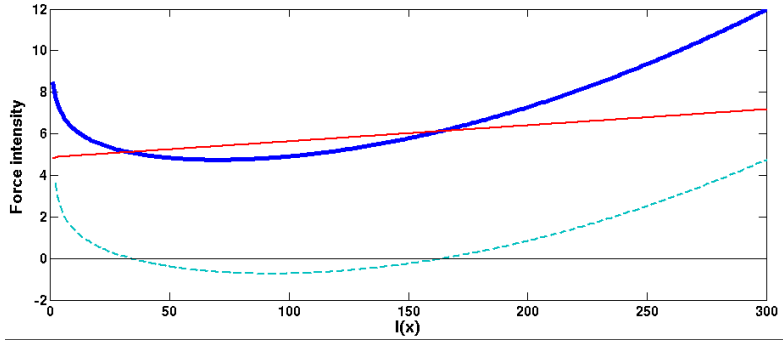

Fig. 2. $F_{\text {data }}(I(x), 70,1 / 130)$ evaluation. The blue thick line corresponds to $-\log \left(f_{i}(I(x), 70)\right.$, the red plain line to $-\log \left(f_{e}(I(x), 1 / 130)\right)$ and the green dotted line to $F_{\text {data }}=$ $-\log \left(f_{i}(I(x), 70)+\log \left(f_{e}(I(x), 1 / 130)\right)\right.$.

around the zero level, an artificial curvature-driven evolution moves the model away from the correct boundaries. After reinitialization, the model goes back to the correct boundaries. Values of the estimated distribution parameters present the same oscillatory behavior which is detected to stop the model. The number of iterations needed to reach this state depends on the data. When the distributions are well differentiated $(\lambda=1 / 30$ and $\sigma=70)$, about 100 iterations were needed, while about 1000 iterations were necessary in the more difficult case $(\lambda=1 / 130$ and $\sigma=70)$. Correct classification rates were evaluated for the two simulated datasets, corresponding to the average of the correct classification rate obtained over the different initializations mentioned above. This rate is $99.4 \%$ when $\lambda=1 / 30$ and $94.7 \%$ when $\lambda=1 / 130$. As expected, correct classification rate was higher when the distributions differentiation is clearer.

\subsection{Results on clinical data}

The segmentation method was tested on four 3D ultrasound datasets acquired with a iU22 (Philips, Eindhoven, Netherlands) and good qualitative results were obtained. Quantitative results are presented for one 3D dataset, using the manual segmentation result previously used for the simulated data generation. The dataset size was $256 \times 256 \times 128$ voxels. Segmentation parameters were set to the same values, and 1500 iterations were needed to reach the oscillatory state. Considering the whole dataset $72 \%$ of the pixels were correctly classified.

Figure 3 presents results on a central slice of this dataset and a $3 \mathrm{D}$ view of the result (a part of the uterus was cropped for visualization purpose). On the classification error image, light gray pixels correspond to amniotic fluid classified as fetal and maternal tissues, and dark gray pixels correspond to the opposite situation.

Image properties can explain the different phenomena leading to a wrong classification. Firstly, the interface between the amniotic fluid and the tissues is blurred, and manual segmentation is not precise at the pixel level, leading to local differences between the two results. Secondly, the interpolation process involved in the volume reconstruction from the acquisition geometry to a Cartesian grid introduces artificial values, generating spatially coherent pixels clusters with high intensity. This explains why pixels between the fetus legs are misclassified. Thirdly, when a structure is highly echogeneous the response of the tissues located behind this structure dramatically falls. This is why brain pixels, located behind the skull, have a very low intensity and are classified as belonging to the amniotic fluid. Fourthly, the regularization term used to obtain 
a smooth result prevents the model to penetrate concavities. Classification errors are consequently present in the contact zone between the fetus arm and the fetus head. Fifthly, pixel intensity of tissues far from the transducer is low because the residual signal is not totally compensated by total gain compensation. Corresponding pixels, belonging to the maternal tissues, are in the same way misclassified as belonging to the amniotic fluid.

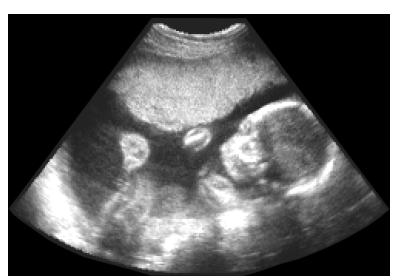

(a)

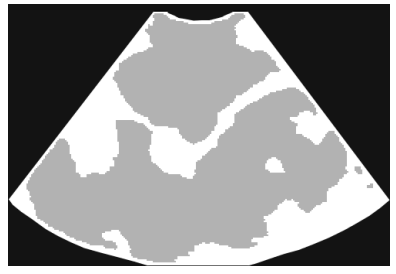

(c)

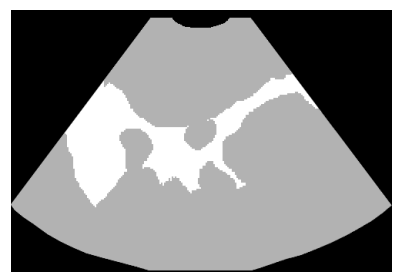

(b)

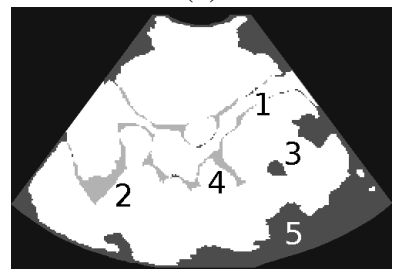

(d)

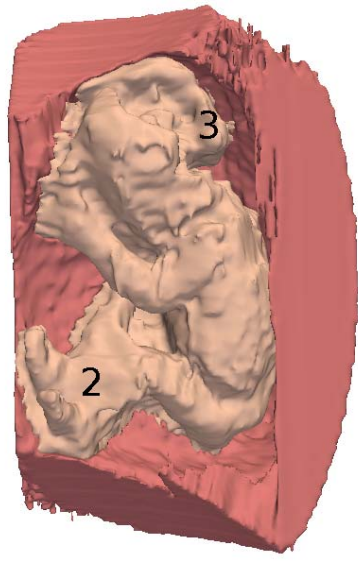

(e)

Fig. 3. (a) Central axial slice of the original dataset. (b-c) Classification results obtained respectively by manual tracing and with the statistical deformable model. White (resp. light gray) pixels correspond to the amniotic fluid (resp. fetal and maternal tissues). (d) Difference between the two classifications (see text). (e) 3D view of the result.

The first three phenomena are inherent to ultrasound acquisitions. For the moment, we have not led further experiments or tested postprocessing methods, which could improve the image quality. Considering the fourth issue, smaller values of $\mu$ were tested. However, as $\mu$ decreases, results become noisy and isolated components appear. The value $\mu=1.2$ seemed to be a good compromise. Segmentation of concavities remains a challenging problem. Considering the fifth issue, we evaluated the correct classification rate in a restricted region. This region corresponds to a dilation of the uterus segmentation, so that structures of interest (the amniotic fluid, the fetus and the uterus border) are included in the region, while pixels located far from the transducer are excluded. In these conditions, the correct classification rate raised to $89 \%$.

Tests were performed, modeling the amniotic fluid response with a Rayleigh distribution. Considering the entire volume and the restricted region, the correct classification rates were respectively $61 \%$ and $67 \%$, inferior to those we have obtained using an exponential distribution to model the response of the amniotic fluid, showing that this distribution is more suitable for this purpose.

\section{CONCLUSION}

We have presented in this paper a methodological framework to embed statistical priors on intensity distributions into a variational minimal partitioning approach. This framework was used to separate the amniotic fluid from the fetal and maternal tissues in 3D fetal ultrasound data. An original modeling of the amniotic fluid response in fetal ultrasound acquisitions was proposed using an exponential distribution, while the fetal and maternal tissues were modeled using the classical Rayleigh distribution. Experiments were led on simulated data, in order to validate the segmentation framework in ideal conditions, with simple parameter and initialization tuning. Due to the particular nature of the data fidelity term combining different probability density function, reinitialization of the $\phi$ function was needed to prevent inappropriate behavior of the curvature term. Good results were obtained on two different simulated datasets. Considering real data, several tests were performed, but only one dataset was analyzed quantitatively, which does not allow us to draw any general conclusion. However, promising results were obtained, especially when considering a region including only the structures of interest.

\section{REFERENCES}

[1] P.W. Callen, Ultrasonography in obstetrics and gynecology, Saunders Philadelphia, 2000.

[2] J.A. Noble and D. Boukerroui, "Ultrasound image segmentation: a survey," IEEE Transactions on Medical Imaging, vol. 25, no. 8, pp. 987-1010, 2006.

[3] S. Osher and J.A. Sethian, Fronts Propagating with Curvature Dependent Speed: Algorithms Based on Hamilton-Jacobi Formulations, National Aeronautics and Space Administration, Langley Research Center, 1987.

[4] Y.C. Chang, T.Y. Hou, B. Merriman, and S. Osher, "A Level Set Formulation of Eulerian Interface Capturing Methods for Incompressible Fluid Flows," Journal of Computational Physics, vol. 124, no. 2, pp. 449-464, 1996.

[5] A. Sarti, C. Corsi, E. Mazzini, and C. Lamberti, "Maximum likelihood segmentation of ultrasound images with Rayleigh distribution," IEEE Transactions on Ultrasonics, Ferroelectrics and Frequency Control, vol. 52, no. 6, pp. 947-960, 2005.

[6] G. Aubert and L. Vese, "A Variational Method in Image Recovery," SIAM Journal on Numerical Analysis, vol. 34, no. 5, pp. 1948-1979, 1997.

[7] C. Gout, C. Le Guyader, and L. Vese, "Segmentation under geometrical conditions using geodesic active contours and interpolation using level set methods," Numerical Algorithms, vol. 39, no. 1, pp. 155-173, 2005.

[8] T.F. Chan and L.A. Vese, "Active contours without edges," IEEE Transactions on Image Processing, vol. 10, no. 2, pp. 266-277, 2001. 